\title{
The vertical vein: To ligate or not to ligate
}

\author{
James S. Tweddell, MD
}

See related article on page 1286.
From the Children's Hospital of Wisconsin, Milwaukee, Wis.

Received for publication Sept 16, 2006; accepted for publication Sept 28, 2006.

Address for reprints: James S. Tweddell, MD, Children's Hospital of Wisconsin, Department of Cardiothoracic Surgery, 9000 W. Wisconsin Ave, MS 715, Milwaukee, WI 53226.

J Thorac Cardiovasc Surg 2007;133:1135-6 $0022-5223 / \$ 32.00$

Copyright () 2007 by The American Association for Thoracic Surgery

doi:10.1016/j.jtcvs.2006.09.116
$\mathrm{T}$ his issue of The Journal of Thoracic and Cardiovascular Surgery includes a contribution from the All India Institute of Medical Sciences summarizing a large experience with total anomalous pulmonary venous connection (TAPVC) that tests a protocol for selective ligation of the vertical vein. ${ }^{1}$ After weaning from cardiopulmonary bypass, the patients underwent test occlusion of the vertical vein, and if the pulmonary artery pressure increased to systemic or suprasystemic levels, the vertical vein was not ligated. The goal was to limit hemodynamic decompensation in the early postoperative period and therefore improve survival. Several important observations are reported. With vertical vein ligation both pulmonary artery and left atrial pressures increased, whereas with restoration of vertical vein patency, in addition to a reduction in pulmonary artery pressure and left atrial pressure, mean arterial pressure increased and acidosis resolved. This suggests a real impact on cardiac output. During pulmonary hypertensive events, which were frequent in this series (40\% of patients), left-to-right shunting was observed in the vertical vein and interpreted by the authors as beneficial. After repair, a patent vertical vein is a connection between the pulmonary venous atrium and the systemic venous system; it functions like an atrial septal defect. The authors also intentionally left an additional atrial septal communication in patients with obstructed TAPVC. The atrial septal defect was left to permit right-to-left shunting during pulmonary hypertensive crises.

An intentional atrial septal communication is commonly created for impaired right-sided heart function, and the benefits are easy to understand; systemic output is maintained, albeit at the expense of some systemic desaturation. This concept is widely applied to patients with a variety of diagnoses, such as tetralogy of Fallot, pulmonary atresia with intact septum, and Ebstein's anomaly. Nevertheless, despite the authors' careful observations, it is difficult to see how vertical vein patency could be of benefit to patients with TAPVC, who are known to have small noncompliant left-sided chambers that result in elevated filling pressures. How would cardiac output be improved by removing preload from the left side? Furthermore, a pulmonary hypertensive crisis results from constriction of pulmonary arteriolar smooth muscle. The result is, among other things, a preload starved left ventricle with critical reduction in cardiac output. How would a left-to-right shunt at the atrial level, which exacerbates preload starvation of the left ventricle, be of any benefit?

Revisiting some observations made during the early years of cardiology and cardiac intervention may provide some insight. A situation somewhat, but admittedly not perfectly, analogous to the physiology observed early after repaired TAPVC is acquired mitral stenosis. In 1916 Lutembacher $^{2}$ described the impact of an atrial septal defect on acquired mitral stenosis. At least initially there was the suggestion that the atrial septal communication limited the impact of mitral stenosis, perhaps by trading mild elevation of right atrial pressure and pulmonary overcirculation for acute, extreme elevation of left atrial pressure. Over time, individuals with mitral stenosis are unquestionably negatively impacted by an atrial septal defect, which increases the left-to-right shunt and predisposes the patient to atrial fibrillation and right ventricular failure. In 1949 Bland and Sweet ${ }^{3}$ described the creation of an atrial level shunt in patients with critical mitral stenosis by anastomosing the azygous vein to a pulmonary vein. The goal was to improve the functional status of patients with critical rheumatic mitral stenosis, permitting the "spill-over" or pop-off of the congested pulmonary veins into the "less tense and 
more capacious systemic venous circulation." 4 The authors hypothesized that, with impaired left ventricular filling, a point is reached where further elevation of left atrial pressure does not result in increased preload to the left ventricle. The patient is symptomatic as a result of the greatly increased left atrial pressure, and the systemic venous system can be used to accommodate this volume. The result is a decrease in left atrial pressure without a decrease in leftsided heart output, albeit at the expense of increased right atrial pressure and increased pulmonary blood flow. Bland and Sweet's experiment resulted in symptomatic improvement, but the experience was small and future efforts were directed at the mitral valve itself and heralded the beginning of cardiac surgery. The observations of Chowdury and colleagues, ${ }^{1}$ Lutembacher, ${ }^{2}$ Bland, ${ }^{4}$ and Cope and colleagues ${ }^{5}$ suggest that the systemic venous system and pulmonary vasculature can accommodate the excess volume and limit the impact of excessive left atrial pressure. Nevertheless, any improvement must be short-lived. Eventually, with continued shunting, the capacity of the systemic venous system and the pulmonary vasculature will be overwhelmed, right atrial pressures will increase, left atrial pressures will return to preintervention levels, and rightsided heart failure will develop with the added stresses of an increased pulmonary to systemic flow ratio. In essence, it is similar to taking the strain off a failing dam by pumping the water from the reservoir back upstream; eventually, the water will return, testing the dam's limits once more.

The authors' observations that acute vertical vein ligation results in greatly elevated left atrial pressure and that left ventricular function and cardiac output are negatively impacted suggests that for a period of time the small, poorly compliant left ventricle of the patient with TAPVC is easily overwhelmed. Indeed, it is routine to see elevated left atrial pressure and pulmonary artery pressure combined with systemic hypotension and evidence of decreased cardiac output after weaning from cardiopulmonary bypass in patients with TAPVC. The postoperative course targets management of these issues, and occasionally extracorporeal membrane oxygenation support is necessary while the pulmonary vascular bed recovers and left ventricular compliance improves. The time course of these adjustments is variable, but commonly the patient with TAPVC with obstruction will have acceptable hemodynamics, albeit sedated, paralyzed, and ventilated, shortly after arrival in the intensive care unit.

Is it possible that the authors have identified a feedback loop whereby acute elevation of left atrial pressure results in excessive pulmonary arteriolar constriction with an out-ofproportion pulmonary hypertensive response that further exacerbates the low cardiac output state caused by a poorly compliant left ventricle? Would patients benefit from a more gradual ligation of the vertical vein so that acute elevation of the left atrial pressure is avoided, albeit at the expense of some period of pulmonary overcirculation and elevated right-sided filling pressure? Unfortunately, the current study design cannot answer that question. To properly test the hypothesis that "vertical vein ligation results in inferior outcome," a prospective randomized trial of ligation versus nonligation would be necessary; otherwise, the authors might be responding to short-lived changes that do not have an impact on survival or to uncontrolled factors, such as age, that are stronger predictors of outcome. The call for a randomized prospective study is the last refuge for those who cannot accept the findings of a study that conflict with their understanding of pathophysiology; furthermore, the rarity and spectrum of TAPVC would make such a trial difficult. Nevertheless, there are problems with adopting the authors' recommendations. The patent vertical vein resulted in right-sided heart failure in approximately half $(11 / 23)$ of the survivors. This is a high complication rate for an unproven therapy. The two groups differed significantly with respect to age. Patients in the ligated vertical vein group were older and had a higher mortality $(29.1 \%)$. The increased mortality in these older patients was more likely the result of the development of pulmonary hypertension rather than the use of vertical vein ligation. Indeed, even patients with obstructed TAPVC appeared to be older than those commonly encountered in North America, and the small number of patients $(2 / 58)$ with infracardiac TAPVC suggests significant presurgical attrition in this group. Finally, it is difficult to reconcile the use of both an atrial septal defect and a persistent vertical vein, which are physiologically equivalent. How can we expect two atrial level communications, the persistent vertical vein and the atrial septal defect, to shunt in different directions in response to the same hemodynamic event? At present the best outcomes for patients with TAPVC are achieved with early diagnosis and timely, accurate repair with minimal manipulation of the pulmonary veins, vertical vein ligation, and careful postoperative management targeting maintenance of cardiac output.

\section{References}

1. Chowdhury UK, Subramaniam G, Joshi K, Varshney S, Kumar G Singh R, et al. Rechanneling of total anomalous pulmonary venous connection with or without vertical vein ligation: results and guidelines for candidate selection. J Thorac Cardiovasc Surg. 2007;133:1286-94

2. Lutembacher R. De la sténose mitrale avec communication interauriculaire. Arch Mal Coeur. 1916;9:237-60.

3. Bland EF, Sweet RH. A venous shunt for marked mitral stenosis. JAMA 1949;140:1259.

4. Bland EF. Rheumatic fever: the way it was. Circulation. 1987;76: 1190-5.

5. Cope JT, Banks D, McDaniel NL, et al. Is vertical vein ligation necessary in repair of total anomalous pulmonary venous connection? Ann Thorac Surg. 1997;64:23-8. 